\title{
artigo
}

Pereira, V.S.; Souza, C.A.; Louro, T.Q.; Oliveira, E.S.; Lima D.M.; Silva, R.C.L.; Marta, C.B.;

Avaliação de tecnologias em saúde: Estado da arte

\section{Avaliação de Tecnologias em Saúde: Estado da Arte}

\begin{abstract}
RESUMO
Objetivos: Realizar uma revisão de literatura sistematizada a respeito da avaliação em tecnologia em saúde e discutir sobre a utilização das avaliações em tecnologias em saúde na tentativa de comparar métodos de análise tradicionais e avaliação em tecnologias em saúde. Metodologia: O presente estudo é caracterizado como revisão sistemática. Os critérios de inclusão adotados para a busca e seleção das publicações foram: artigos publicados em periódicos científicos nacionais e internacionais que abordassem a temática, no período de 2010 a 2018. Resultados: Foram encontrados 31 artigos. Os artigos foram distribuidos em três categorias: Avaliação de tecnologia em saúde auxiliando na tomada de decisão. Participação de profissionais que utilizam a tecnologia para a avaliação de tecnologia em saúde e Comitê permanente de avaliação em tecnologia. Conclusão: Ao utilizar evidências clínicas para a avaliação em tecnologia em saúde existe a possibilidade de avaliação do custo-efetividade em comparação aos métodos tradicionais de análise.
\end{abstract}

DESCRITORES: Avaliação da Tecnologia Biomédica; Gestão em Saúde; Enfermagem.

\section{ABSTRACT}

Objectives: Conduct a systematized literature review on health technology assessment and discuss the use of health technology assessment to compare traditional analysis methods and health technology assessment. Methodology: This study is characterized as a systematic review. The inclusion criteria adopted for the search and selection of publications were articles published in national and international scientific journals that addressed the theme, from 2010 to 2018. Results: Were found 31 articles. The articles were divided into three categories: Health technology assessment assisting in decision making. Participation of professionals who use technology to evaluate such technology and the Permanent Technology Assessment Committee. Conclusion: By using clinical evidence for health technology assessment there is a possibility of cost-effectiveness assessment compared to traditional methods of analysis.

KEYWORDS: Technology Assessment, Biomedical; Health Management; Nursing.

\section{RESUMEN}

Objetivos: Realizar una revisión sistemática de la literatura sobre evaluación de tecnología de salud y discutir el uso de la evaluación de tecnología de salud en un intento de comparar los métodos de análisis tradicionales y la evaluación de tecnología de salud. Metodología: este estudio se caracteriza por ser una revisión sistemática. Los criterios de inclusión adoptados para la búsqueda y selección de publicaciones fueron: artículos publicados en revistas científicas nacionales e internacionales que abordaron el tema, de 2010 a 2018. Resultados: Encontramos 31 artículos. Los artículos se dividieron en tres categorías: Evaluación de tecnología de salud que ayuda en la toma de decisiones. Participación de profesionales que usan tecnología para evaluar dicha tecnología y el Comité Permanente de Evaluación de Tecnología. Conclusión: Al usar evidencia clínica para la evaluación de la tecnología de salud, existe la posibilidad de una evaluación de costo-efectividad en comparación con los métodos tradicionales de análisis.

PALABRAS CLAVE: Evaluación de la Tecnología Biomédica; Gestión en Salud; Enfermería.

RECEBIDO EM: 11/11/2019 APROVADO EM: 12/11/2019

\section{Vinícius Sidney Pereira}

Pós-Graduando de Enfermagem em Alta Complexidade pela Universidade Veiga de Almeida. Enfermeiro assistencial do CTI adulto do Hospital Casa Rio Laranjeiras.

\section{Camilla Alves de Souza}

Enfermeira especialista em Saúde da Família pela (SMS-RJ/ Unigranrio). Clínica da Família Heitor dos Prazeres (SMS/RJ). 


\section{Thiago Quinellato Louro}

Pós-Doutor. Professor Adjunto na Universidade Federal Fluminense.

\section{Elson Santos de Oliveira}

Doutorando em Enfermagem e Biociência. Professor Assistente do Curso de Graduação de Enfermagem na Universidade Veiga de Almeida; Coordenador da Pós-Graduação de Enfermagem em Alta Complexidade da Universidade Veiga de Almeida; Enfermeiro assistencial do CTI Adulto do INCa.

\section{Daiana Miranda Lima}

Especialista em Enfermagem Neonatal pelo IFF/Fiocruz e Mestre em Enfermagem pela Unirio. Servidora no Hospital Universitário Gaffrée e Guinle.

\section{Roberto Carlos Lyra da Silva}

Doutor em Enfermagem. Professor Associado III. Coordenador do Programa de Pós-Graduação em Enfermagem e Biociências. Universidade Federal do Estado do Rio de Janeiro.

\section{Cristiano Bertolossi Marta}

Pós-Doutor em Enfermagem com ênfase em Avaliação. Professor Adjunto II do Departamento de Fundamentos de Enfermagem da Faculdade de Enfermagem da UERJ. Diretor de Pesquisa e Coordenador Geral das Pós-graduações em Enfermagem da Universidade Veiga de Almeida.

\section{INTRODUÇÃO}

A saúde é um dos setores que mais gera inovação em serviços e produtos, acarretando elevados gastos, os quais nem sempre compensam a aquisição de novas mercadorias e serviços a médio e longo prazos.

Ao mesmo tempo, gestores são pressionados para que tecnologias novas e emergentes sejam incorporadas, segundo Roberto $^{(1)}$. Tendo em vista essa realidade, torna-se indispensável estudo criterioso fundamentado nas evidências científicas, nas questões operacionais, econômicas e técnicas para que a tomada de decisão seja eficiente e sustentável.

$\operatorname{Medeiros}^{(2)}$ cita que a Avaliação de Tecnologias em Saúde (ATS) obteve avanços expressivos na condução das decisões relacionadas à incorporação de novas tecnologias nos sistemas de saúde. Contudo, há uma série de impedimentos quando se trata de avaliar tecnologias que já estão sendo oferecidas há décadas, muitas sequer tiveram sua eficácia e segurança comprovadas e ainda geram gastos expressivos, quando comparadas às novas alternativas que estão surgindo.

Executar simples comparações técnicas de desempenho não é suficiente para produtos que abrigam alta tecnologia, também é importante medir o potencial ganho clínico que está agregado a essa inovação.
De acordo com Lourenço ${ }^{(3)}$, a melhora no serviço está relacionada com o gerenciamento de custos, sendo assim, uma preocupação importante é a alocação inadequada de recursos em saúde em áreas de maior custo e benefício clínico duvidoso.

Para a prática, o estudo justifica-se dando maior visibilidade às ações das avaliações em tecnologias, podendo auxiliar na tomada de decisão dos gestores na incorporação de tecnologias com maior custo-benefício em suas instituições. Para a pesquisa, o estudo justifica-se pelo tema emergente, além da necessidade de elucidar o hiato da literatura existente em relação a avaliação de tecnologias em saúde.

Neste sentido, a questão dessa pesquisa é: $\mathrm{O}$ uso de avaliação em tecnologia é mais custo efetivo quando comprado com métodos tradicionais de tomada de decisão pelo gestor? O estudo tem como objetivos realizar uma revisão de literatura sistematizada a respeito da avaliação em tecnologia em saúde e discutir sobre a utilização das avalições em tecnologias em saúde na tentativa de comparar métodos de análise tradicionais e avaliação em tecnologias em saúde.

\section{METODOLOGIA}

O presente estudo é caracterizado como revisão sistemática que, de acordo com Sampaio ${ }^{(4)}$, utiliza a literatura como fonte de dados, sendo útil para agregar informações de um apanhado de estudos incorporando uma visão maior de resultados, podendo auxiliar avaliações futuras e ampliando as conclusões das informações encontradas. Este artigo se propõe a trazer uma síntese, em termos teóricos e empíricos baseada na análise de conteúdo desenvolvida por Bardin ${ }^{(5)}$.

Os critérios de inclusão adotados para a busca e seleção das publicações foram: artigos publicados em periódicos científicos nacionais e internacionais que abordassem a temática: avaliação de tecnologias em saúde na compra de insumos ou serviços, nas línguas portuguesa, inglesa e espanhola, no período de 2010 a 2018 e indexados nas bases de dados: Literatura Latino-Americana e do Caribe em Ciências da Saúde (LILACS); Índice Bibliográfico Espanhol de Ciências da Saúde (IBECS), Base de Dados de Enfermagem (BDENF), Medical Literature Analysis and Retrieval System Online (MEDLINE) e na biblioteca Scientific Electronic Library Online (SciELO), artigos disponibilizados na íntegra, diretamente pelo site da base ou pelo portal Capes, localizáveis por intermédio da combinação dos seguintes descritores cadastrados no Portal de Descritores das Ciências da Saúde (DeCS): "Avaliação em tecnologias biomédicas", "Gestão em saúde", "Análise Custo-Benefício", "Avaliação 


\section{artigo}

Pereira, V.S.; Souza, C.A.; Louro, T.Q.; Oliveira, E.S.; Lima D.M.; Silva, R.C.L.; Marta, C.B.

Avaliação de tecnologias em saúde: Estado da arte

de Custo-Efetividade", "Custo e Análise de Custo", "Custos hospitalares" e "Redução de Custos". Esses descritores foram combinados com os operadores booleanos "AND" e "OR" a fim de refinar os estudos de acordo com o tema em questão.

A busca foi realizada de forma ordenada e foram utilizados como critérios de exclusão: artigos fora do período considerado, artigos que não disponi-

Fluxograma 1. Identificação, seleção e inclusão dos artigos investigados. Rio de Janeiro, RJ Brasil, 2018

Artigos identificados na base de dados ( $N=19.819)$

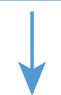

MEDLINE

$(2=6262)$
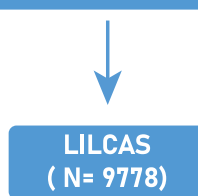

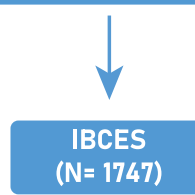

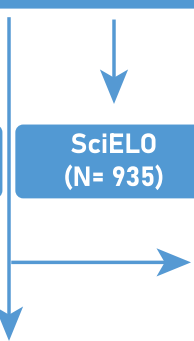

Artigos selecionados após a primeira análise ( $\mathrm{N}=\mathbf{5 0 0})$

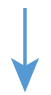

Artigos selecionados após a leitura do título e resumo (N=31)

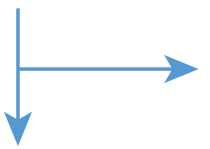

artigos excluído após leitura na integra $(\mathrm{N}=14)$ bilizavam o texto completo e publicados em mais de uma base (duplicatas). Após a seleção dos artigos, realizou-se a leitura criteriosa do título e do resumo de cada publicação a fim de verificar a consonância com a pergunta norteadora da investigação. Quando houve dúvida referente à inclusão ou exclusão do estudo, ele foi lido na íntegra para reduzir o risco de perdas de publicações relevantes ao estudo. Desse modo, aqueles que não abordaram o tema "Avaliação em tecnologias em saúde" foram descartados na segunda análise.

A coleta de dados se deu entre os meses de agosto e outubro de 2018 e contou com o apoio de um instrumento de coleta de dados elaborado no software Microsoft Office Word $2019^{\circ}$, com as seguintes variáveis: título do artigo, autores, periódico, ano de publicação, tipo/abordagem do estudo.

\section{RESULTADOS}

Foram selecionados 31 artigos, destes, 17 abordavam avaliação de tecnologias em saúde e foram selecionados para o estudo de acordo com os critérios de inclusão e exclusão estabelecidos. A seleção final dos artigos é apresentada no Quadro 1.

Quadro 1. Artigos selecionados para análises. Rio de Janeiro, RJ, Brasil, 2018

\begin{tabular}{|l|c|c|c|c|c|}
\hline TíTULO DO ARTIGO/ESTADO DE PUBLICAÇÃo & AUTORES & ANO & TIPO DE ESTUDO & PERIÓDICO & $\begin{array}{c}\text { ANO DE } \\
\text { COLETA }\end{array}$ \\
\hline $\begin{array}{l}\text { Avanços e desafios da Política Nacional de Gestão } \\
\text { de Tecnologias em Saúde - Distrito Federal (BR) }\end{array}$ & $\begin{array}{c}\text { Silva HP, } \\
\text { Petramal CA, } \\
\text { Elias F S. }\end{array}$ & 2012 & Qualitativo & $\begin{array}{c}\text { Rev. Saúde } \\
\text { Pública }\end{array}$ & 2018 \\
\hline $\begin{array}{l}\text { Gestão de material médico-hospitalar e o } \\
\text { processo de trabalho em um hospital público } \\
\text { - Distrito Federal (BR) }\end{array}$ & $\begin{array}{c}\text { Garcia SD, et } \\
\text { al. }\end{array}$ & 2012 & Qualitativo & $\begin{array}{c}\text { Rev. Bras } \\
\text { Enferm }\end{array}$ & 2018 \\
\hline $\begin{array}{l}\text { Participação efetiva do enfermeiro no planeja- } \\
\text { mento: foco nos custos - Distrito Federal (BR) }\end{array}$ & $\begin{array}{c}\text { Albano TC, } \\
\text { Freitas J B. }\end{array}$ & 2013 & Quantitativo & $\begin{array}{c}\text { Rer. Bras } \\
\text { Enfermagem }\end{array}$ & 2018 \\
\hline $\begin{array}{l}\text { Análise das Reprovações de Materiais Médico- } \\
\text {-Hospitalares de um Hospital de Ensino - Minas } \\
\text { Gerais (BR) }\end{array}$ & $\begin{array}{c}\text { Reis L B, et al. } \\
\text { Q }\end{array}$ & 2015 & Quantitativo & $\begin{array}{c}\text { Revista de } \\
\text { administração } \\
\text { hospitalar e } \\
\text { inovação em } \\
\text { saúde }\end{array}$ & 2018 \\
\hline $\begin{array}{l}\text { Análise de impacto orçamentário: uma revisão } \\
\text { prática de conceitos e aplicações para o gestor - } \\
\text { São Paulo (BR) }\end{array}$ & $\begin{array}{c}\text { Medeiros M F } \\
\text { B, et al }\end{array}$ & 2018 & Qualitativo & $\begin{array}{c}\text { J. Bras } \\
\text { Economia em } \\
\text { Saúde }\end{array}$ & 2018 \\
\hline
\end{tabular}




\begin{tabular}{|c|c|c|c|c|c|}
\hline $\begin{array}{l}\text { Avaliação e incorporação de tecnologias em } \\
\text { saúde: processo e metodologia adotados por } \\
\text { um hospital universitário de alta complexidade } \\
\text { assistencial - Rio de Janeiro (BR) }\end{array}$ & Nunes AA. et al & 2013 & Qualitativo & $\begin{array}{l}\text { Cad. Saúde } \\
\text { Pública }\end{array}$ & 2018 \\
\hline $\begin{array}{l}\text { Avaliação de Tecnologias em Saúde: Contexto } \\
\text { Histórico e Perspectivas - Distrito Federal (BR) }\end{array}$ & $\begin{array}{l}\text { Amorim FF, } \\
\text { Ferreira JR } \\
\text { PN, Faria ER, } \\
\text { Alameida KJQ. }\end{array}$ & 2011 & Qualitativo & $\begin{array}{l}\text { Com. Ciências } \\
\text { Saúde }\end{array}$ & 2018 \\
\hline $\begin{array}{l}\text { Estudo de custo-análise do tratamento da artri- } \\
\text { te reumatoide grave em um município do Sul do } \\
\text { Brasil - Rio de Janeiro (BR) }\end{array}$ & $\begin{array}{l}\text { Buendgens FB, } \\
\text { et al. }\end{array}$ & 2013 & Qualitativo & $\begin{array}{l}\text { Cad. Saúde } \\
\text { Pública }\end{array}$ & 2018 \\
\hline $\begin{array}{l}\text { O Gestor Hospitalar e Sua Atuação Frente ao } \\
\text { Suprimento de Materiais - Rio de Janeiro (BR) }\end{array}$ & $\begin{array}{l}\text { Roberto WLC, } \\
\text { Lira RA. }\end{array}$ & 2010 & Qualitativo & $\begin{array}{l}\text { Perspectivas } \\
\text { Online }\end{array}$ & 2018 \\
\hline $\begin{array}{l}\text { Incorporação tecnológica no contexto da saúde: } \\
\text { uma análise de custo efetividade das bombas de } \\
\text { infusão na insulinoterapia intravenosa - Rio de } \\
\text { Janeiro (BR) }\end{array}$ & Moreira APA. & 2017 & Quanti-qualitativo & UNIRIO & 2018 \\
\hline $\begin{array}{l}\text { O papel da avaliação de tecnologias em saúde } \\
\text { (ATS) na retirada de dispositivos médicos obso- } \\
\text { letos no Sistema Único de Saúde (SUS) - Rio de } \\
\text { Janeiro (BR) }\end{array}$ & Assis EC. & 2013 & Qualitativo & ENSP & 2018 \\
\hline $\begin{array}{l}\text { Organizações de avaliação de tecnologias em } \\
\text { saúde (ATS): dimensões do arcabouço institucio- } \\
\text { nal e político - Rio de Janeiro (BR) }\end{array}$ & $\begin{array}{l}\text { Novaes HMD, } \\
\text { Soarez PC. }\end{array}$ & 2016 & Qualitativo & $\begin{array}{l}\text { Cad. Saúde } \\
\text { Pública }\end{array}$ & 2019 \\
\hline $\begin{array}{l}\text { Perfil de Estudos da Rede Brasileira de Avaliação } \\
\text { de Tecnologia em Saúde (Rebrats) de } 2004 \text { a } \\
2015 \text { - Distrito Federal (BR) }\end{array}$ & Elias FTS, et al. & 2016 & Qualitativo & $\begin{array}{l}\text { Com. Ciências } \\
\text { Saúde }\end{array}$ & 2019 \\
\hline $\begin{array}{l}\text { Uso da avaliação de tecnologias em saúde em } \\
\text { processos de análise para incorporação de } \\
\text { tecnologias no Sistema Único de Saúde no } \\
\text { Ministério da Saúde - Rio de Janeiro (BR) }\end{array}$ & $\begin{array}{l}\text { Novaes HM, } \\
\text { Elias FTS. }\end{array}$ & 2013 & Qualitativo & $\begin{array}{l}\text { Cad. Saúde } \\
\text { Pública }\end{array}$ & 2019 \\
\hline $\begin{array}{l}\text { Tecnologias em saúde: proposição de um time } \\
\text { de terapia intravenosa na unidade de terapia } \\
\text { intensiva - Rio de Janeiro (BR) }\end{array}$ & Moreira APA. & 2012 & Qualitativo & UFF & 2019 \\
\hline $\begin{array}{l}\text { Participação do enfermeiro na gestão de recur- } \\
\text { sos hospitalares - Distrito Federal (BR) }\end{array}$ & $\begin{array}{l}\text { Ventura PFEV, } \\
\text { Freire EMR, } \\
\text { Alves M. }\end{array}$ & 2016 & Qualitativo & $\begin{array}{c}\text { Revista } \\
\text { Eletrônica } \\
\text { Gestão \& Saúde }\end{array}$ & 2019 \\
\hline $\begin{array}{l}\text { Análise de custo-efetividade do reúso de catete- } \\
\text { res de cinecoronariografia sob a perspectiva de } \\
\text { uma instituição pública no Município do Rio de } \\
\text { Janeiro, Brasil - Rio de Janeiro (BR) }\end{array}$ & $\begin{array}{l}\text { Veras BMG, } \\
\text { et al. }\end{array}$ & 2013 & Qualitativo & $\begin{array}{l}\text { Cad. Saúde } \\
\text { Pública }\end{array}$ & 2019 \\
\hline
\end{tabular}

\section{DISCUSSÃO}

Os artigos foram distribuidos em três categorias: Avaliação de tecnologia em saúde auxiliando na tomada de decisão, Participação de profissionais que utilizam a tecnologia para a avaliação de tecnologia em saúde e Comitê permanente de avaliação em tecnologia.
Avaliação de tecnologia em saúde auxiliando na tomada de decisão pelo gestor

De acordo com Nunes ${ }^{(6)}$, a avaliação das tecnologias baseada na rede hospitalar é prática aconselhável mundialmente, garantindo melhor qualidade assistencial e atendendo às necessidades dos usuários.

Para Assis ${ }^{(7)}$, as novas tecnologias, geralmente, têm maior custo e não têm a obrigatoriedade de substituir tecnologias anteriores.
Os gestores podem encontrar dificuldades para a aquisição de novas tecnologias, para isso, necessitam de avaliação de custo-efetividade, impacto orçamentário e custo-oportunidade para serem implementadas.

Ao implementar uma nova tecnologia em saúde, ou no caso de exclusão de alguma tecnologia em saúde, se faz necessário avaliar as evidências científicas sobre tal artigo. Pode-se mencionar que Silva ${ }^{(8)}$, ao falar sobre 
análise e recomendação de tecnologias em saúde, cita que sejam priorizados os resultados clinicamente testados, que tragam benefícios, segurança e custo-efetividade.

Geralmente, as ações em ATS são desenvolvidas em nível central e existem desafios na disseminação dessas ações em níveis meso e micro, além de difundir para instituições privadas de saúde.

Pode-se citar quatro publicaçôes: "Elaboração de Pareceres Técnico-Científicos", "Estudos de Avaliação Econômica de Tecnologias em Saúde", "Manual de Análise de Impacto Orçamentário de Tecnologias em Saúde" e "Proposta de Monitoramento do Horizonte Tecnológico no SUS", com a finalidade de disseminare, de certa forma, padronizar métodos de estudos de ATS. Os estudos podem ser realizados internamente ou por especialistas externos.

De acordo com Silva ${ }^{(8)}$, seria importante para os gestores de intituições privadas de saúde criar mecanismos para que as decisões de inclusão de tecnologias em saúde sejam tomadas com base em evidências clínicas, além de realizar articulação com os grupos de ATS já desenvolvidos dentro e fora do Sistema Único de Saúde (SUS).

\section{Participação de profissionais que utilizam a tecnologia para a avalia- ção de tecnologia em saúde}

As avaliações de tecnologias em saúde realizadas em nível central, ou seja, fora dos complexos assitenciais que mais a utilizam, não acolhem às necessidades e especificidades das organizações de saúde, impactando a prática assistencial.

Os profissionais envolvidos no gerenciamento das unidades hospitalares, sejam eles médicos, enfermeiros, farmacêuticos, dentre outros, devem possuir conhecimentos que possibilitem tomadas de decisões e alocações de recursos.

O profissional precisa desenvolver uma visão holística da assistência em saúde prestada, além de desenvolver certas habilidades gerenciais, habilidades essas que nem sempre são ofertadas na Graduação, sendo assim, o profissional vê a necessidade de procurar conhecimento além da sua formação. Tendo em vista que para a qualidade da assistência as ações gerenciais precisam estar integradas, citando Garcia ${ }^{(9)}$, "a qualidade e quantidade do material é um dos aspectos que garante a eficiência do cuidado prestado".

Albano $^{(10)}$ cita que o profissional enfermeiro tem competência técnica para participar da avalição de tecnologias em saúde pelo seu contato diário com maior variedade de materiais utilizados na prestação dos cuidados aos pacientes/clientes, facilitando a formação de visão crítica sobre o assunto.

A prática diária permite o conhecimento dos materiais empregados para assitência aos pacientes com qualidade e segurança, porém, em algumas situações, os profissionais de saúde não têm autonomia na escolha da tecnologia em saúde, ocorrendo centralização da escolha e das avaliações.

Garcia ${ }^{(9)}$ traz essa conclusão em seu estudo quando diz que a falta de envolvimento dos profissionais pode ser por falta de conhecimento da importância de sua participação e da falta de programas educacionais relacionados ao tema. O que nos mostra a necessidade do fortalecimento do processo de educação continuada.

\section{Comitê permanente de avaliação em tecnologia}

No nível central, existe a Comissão Nacional de Incorporação de Tecnologias no SUS (CONITEC), criada em 2011, que é um órgão colegiado de caráter permantente que assessora o Ministério da Saúde acerca de incorporação, alteração ou exclusão de tecnologias em saúde pelo SUS.

A criação de um comitê permanente de avaliação em tecnologia, em nível meso, pode contribuir para a decisão de inclusão, alteração ou exclusão de determinada tecnologia em um serviço de saúde.

Um comitê permanente de avaliação técnica de materiais e medicamentos, composta por profissionais e usuários finais ou representantes destes, permite avaliar e supervisionar o padrão de qualidade.

A insuficiência de recursos orçamentários, desperdícios, má utilização de insumos e equipamentos, escassa qualificação dos profissionais da área, dente outros, podem levar a problemas de abastecimento de insumos e medicamentos, a criação de um comitê de avaliação de tecnologia em saúde pode possibilitar a identificação de prioridades para incorporação de tecnologias nos sistemas de saúde.

De acordo com Nunes ${ }^{(()}$, um comitê que se reune quinzenalmente e classifica as tecnologias em quatro níves de prioridade e de acordo com os níveis as solicitações de tecnologias são incorporadas ou são realizados estudos mais abrangentes para sua incorporação ou não.

\section{CONCLUSÃO}

Fatores como recursos financeiros limitados, evidências científicas insuficientes e recursos humanos escassos mostram a necessidade de criatividade e empenho para superar esses entraves na utilização de ATS, principalmente, nos níveis micro e meso de decisão acerca da implantação de novas tecnologias em unidades de saúde.

Entende-se que o envolvimento de profissionais de saúde e membros da sociedade, além de discussão com outros países com experiência em uso de ATS, pode contribuir em questões relacionadas à incorporação, avaliação ou exclusão de tecnologias em saúde, tanto no SUS quanto em rede privada de saúde.

Ao utilizar evidências clínicas para a avaliação em tecnologia em saúde, possibilita a avaliação do custo-efetividade em comparação aos métodos tradicionais. A criação de uma comissão de avaliação em tecnologia pode auxiliar na sustentação das decisões gerenciais das organizações de saúde, racionalizando o uso de novas tecnologias.

\section{REFERÊNCIAS}

1. Roberto WLC, Lira RA. O Gestor Hospitalar e Sua Atuação Frente ao Suprimento de Materiais. Perspectivas Online [Internet]. 2010 [acesso em 2018 out 8]; 4(13):87-104. Disponivel em: http://ojs3.perspectivasonline.com.br/index.php/revista_ antiga/article/view/412.

2. Medeiros MFB, et al. Análise de impacto orçamentário: uma revisão prática de conceitos e aplicações para o ges- 


\section{REFERÊNCIAS}

tor. J Bras. Econ. Saúde [Internet]. 2018 [acesso em 2018 out 8]; 10(1):75-79. Disponivel em: http://docs.bvsalud.org/ biblioref/2018/05/884397/jbes101-doi-1021115_jbesv10n1p75-79.pdf.

3. Lourenço KG, Castilho V. Classificação ABC dos materiais: uma ferramenta gerencial de custos em enfermagem. Rev bras enferm [Internet]. 2006 [acesso em 2018 out 8]; 59(1):52-55. Disponivel em: http://www.scielo.br/scielo.php?script=sci_arttext\&pid=S0034-71672006000100010.

4. Sampaio RF, Mancini MC. Estudos de revisão sistemática: um guia para síntese criteriosa da evidência científica. Rev. bras. fisioter. [Internet]. 2007 [acesso em 2018 agosto 15]. Disponivel em: http://www.scielo.br/scielo.php?script=sci_arttext\&pid=S1413-35552007000100013.

5. Bardin L. Análise de conteúdo. 6. ed. Lisboa: Edições 70; 2011.

6. Nunes AA, et al. Avaliação e incorporação de tecnologias em saúde: processo e metodologia adotados por um hospital universitário de alta complexidade assistencial. Cad. Saúde Pública [Internet]. 2013 [acesso em 2018 out 15]; 29(Sup.):179-186. Disponivel em: http://www.scielo.br/pdf/csp/v29s1/a16.pdf.

7. Assis EC. 0 papel da avaliação de tecnologias em saúde (ATS) na retirada de dispositivos médicos obsoletos no Sistema Único de Saúde (SUS) [Dissertação] [Internet]. Rio de Janeiro: Escola Nacional de Saúde Pública Sergio Arouca; 2013 [acesso em 2018 nov. 20]. Disponivel em: http://www6.ensp.fiocruz.br/ visa/files/assisecm_0.pdf.

8. Silva HP, Petramal CA, Elias FS. Avanços e desa os da Política Nacional de Gestão de Tecnologias em Saúde. Rev. Saúde Pública [Internet]. 2012 [acesso em 2018 out 18]; 46(Sup.):83-90. Disponivel em: http://www.scielo.br/scielo.php?script=sci_arttext\&pid=S0034-89102012000700012.

9. Garcia SD, et al. Gestão de material médico-hospitalar e o processo de trabalho em um hospital público. Rev Bras Enferm [Internet]. 2012 [acesso em 2018 out 18]; 65(2):339-346. Disponivel em: http://www.scielo.br/pdf/reben/v65n2/v65n2a21. pdf.

10. Albano TC, Freias JB. Participação efetiva do enfermeiro no planejamento: foco nos custos. Rev Bras Enferm [Internet]. 2013 [acesso em 2018 nov 20]; 66(3):372-377. Disponivel em: http://www.scielo.br/pdf/reben/v66n3/a11v66n3.pdf.

11. Moreira APA. Incorporação tecnológica no contexto da saúde: uma análise de custo efetividade das bombas de infusão na insulinoterapia intravenosa [tese] [Internet]. Rio de Janeiro: Universidade Federal do Estado do Rio de Janeiro; 2017. [acesso em 2018 nov. 20]. Disponivel em http://www2.unirio.br/unirio/ccbs/ppgenfbio/arquivos/teses-arquivos/46-ana-moreira-2017.

12. Mendes KDS, Silveira RCCP, Galvão CM. Revisão integrativa: método de pesquisa para a incorporação de evidências na saúde e na enfermagem. Texto Contexto Enfermagem [Internet]. 2008 [acesso em 2018 out 20]; 17(4):758-764. Disponivel em: http:// www.scielo.br/pdf/tce/v17n4/18.pdf.
13. Reis LB, et al. Análise das Reprovações de Materiais Médico-Hospitalares de um Hospital de Ensino. RAHIS [Internet]. 2015 [acesso 2018 nov 20]; 12(3):10-21. Disponivel em: https://revistas.face.ufmg.br/index.php/rahis/article/view/2432.

14. Amorim FF, Ferreira Junior PN, Faria ER, Almeida KJQ. Avaliação de Tecnologias em Saúde: Contexto Histórico e Perspectivas. Com Ciências Saúde [Internet]. 2011 [acesso em 2018 dez 15]; 21(4):343-348. Disponivel em: http://bvsms.saude.gov.br/ bvs/artigos/avaliacao_tecnologias_saude.pdf

15. Buendgens FB, et al. Estudo de custo-análise do tratamento da artrite reumatoide grave em um município do Sul do Brasil. CadSaúde Pública [Internet]. 2013 [acesso em 2018 dez 15]; 29(Sup):81-91. Disponivel em: http://www.scielo.br/pdf/csp/ v29s1/a08.pdf.

16. Novaes HMD, Soárez PC. Organizações de avaliação de tecnologias em saúde (ATS): dimensões do arcabouço institucional e político. Cad Saúde Pública [Internet]. 2016 [acesso em 2019 jan 10]; 32(Sup2):1-14. Disponivel em: http://www.scielo.br/ pdf/csp/v32s2/pt_1678-4464-csp-32-s2-e00022315.pdf.

17. Elias FTS, et al. Perfil de Estudos da Rede Brasileira de Avaliação de Tecnologia em Saúde (Rebrats) de 2004 a 2015. Com Ciências Saúde [Internet]. 2016 [acesso em 2019 jan 10]; 27(1):53-58. Disponivel em: http://www.escs.edu.br/revistaccs/index.php/comunicacaoemcienciasdasaude/article/ view/88/24.

18. Novaes HM, Elias FTS. Uso da avaliação de tecnologias em saúde em processos de análise para incorporação de tecnologias no Sistema Único de Saúde no Ministério da Saúde. Cad Saúde Pública [Internet]. 2013 [acesso em 2019 jan 20]; 29(Sup):7-16. Disponivel em: http://www.scielo.br/pdf/csp/v29s1/a02.pdf.

19. Instituto de Saúde (BR). Avaliação de Tecnologias e Inovação em Saúde no SUS: Desafios e Propostas para a Gestão. São Paulo: Instituto de Saúde; 2015.

20. Ministério da Saúde (BR). Política Nacional de Gestão de Tecnologias em Saúde. Brasília: Ministério da Saúde; 2010.

21. Moreira APA. Tecnologias em saúde: proposição de um time de terapia intravenosa na unidade de terapia intensiva [dissertação] [Internet]. Rio de Janeiro: Universidade Federal Fluminense; 2012. [acesso em 2019 fev 02]. Disponivel em: https:// app.uff.br/riuff/handle/1/1022.

22. Ventura PFEV. Participação do enfermeiro na gestão de recursos hospitalares [dissertação] [Internet]. Belo Horizonte: Universidade federal do Minas Gerais; 2016. [acesso em 2019 fev 02]. Disponivel em: https://repositorio.ufmg.br/bitstream/1843/GCPA-8GSJKX/1/palloma_fernades_estanislau_ vaz_ventura.pdf.

23. Veras BMG, et al. Análise de custo-efetividade do reúso de cateteres de cinecoronariografia sob a perspectiva de uma instituição pública no Município do Rio de Janeiro, Brasil. Cad Saúde Pública [Internet]. 2013 [acesso em 2019 fev 02]; 29(Sup):110-120. Disponivel em: http://www.scielo.br/pdf/ csp/v29s1/a10.pdf. 